\title{
POPULUS: DESIGN PROCESS, FROM PROTOTYPING TO THE OBJECT
}

\author{
Christian Eugenia Hernández Ruiz \\ Universidad Michoacana de San Nicolás de Hidalgo | México | evgenyruz@gmail.com \\ José Aurelio Espinoza Moreno \\ Universidad Michoacana de San Nicolás de Hidalgo | México | arq.ae.mx@gmail.com
}

\begin{abstract}
Populus it's a design proposal for concrete urban furniture, which is a new interpretation of popular design, helping to solve the shortage of functional equipment that is comfortable, durable and capable of being integrated into multiple contexts. This study reveals the importance of understanding the object before building it to user's scale taking advantage of the tools provided by different methodologies such as rapid prototyping and digital manufacturing to explore material and functional aspects. In addition to validate the usefulness and adaptability of the creative process with the designer's labor in an emerging context such as Latin America.
\end{abstract}

Keywords: Urban furniture; Design process; Popular design; Rapid prototyping; Digital manufacturing.

\section{INTRODUCCIÓN}

En la contemporaneidad, la expansión urbana y la concentración de la población en las ciudades latinoamericanas, han expuesto un constante estado de emergencia y escasez, que propicia un déficit de equipamiento e infraestructura de calidad en el espacio público, evidenciando la obsolescencia de los elementos que lo componen, particularmente del mobiliario urbano.

Por esta razón, es de vital importancia reconocer la naturaleza del mobiliario urbano, entendido como un componente que conforma una estructura más compleja, y que parte de un requerimiento básico de infraestructura y/o equipamiento (Del Real, 2013). El mobiliario urbano se entiende como aquellas micro-arquitecturas u objetos urbanos de uso público, normalmente conformados por: farolas, señalamientos, cestos, vallas, bolardos, y particularmente asientos o bancos, estos últimos son el motivo de estudio del presente trabajo, los cuales son indispensables para la construcción y activación del espacio público de calidad.

En la era moderna del diseño, el mobiliario, y particularmente, la silla ha sido un receptáculo donde se prueban materiales, se perfeccionan técnicas y se comprueban teorías. En el México del S. XX, el diseño de asientos tiene dos vertientes, la primera comienza a trazarse en el entorno urbano y se asocia a los estilos artísticos de principios del siglo; mientras que la segunda proviene de la tradición popular. (Mallet, 2018).

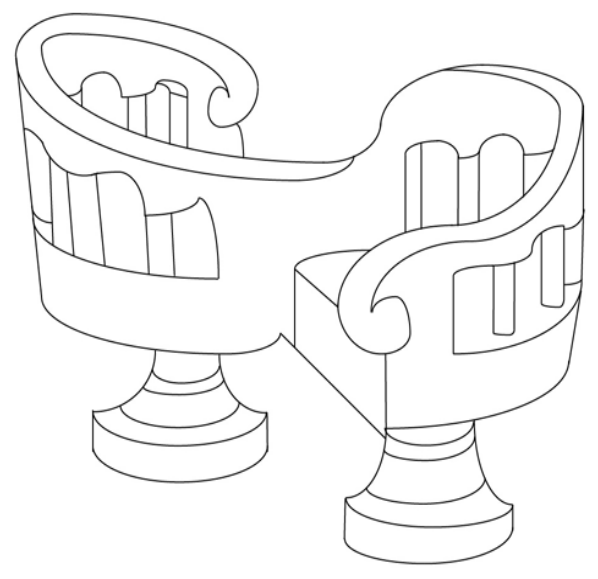

Figura 1: llustración de la Silla Confidentes.

Derivado del contexto anterior, se identifica la "Silla Confidentes" (Figura 1), instaladas por vez primera en la Plaza Grande de Mérida en el año de 1915 (Mendoza, 2016). Hoy en día, apropiada por la gente, caracteriza toda una región de la península de Yucatán. Este ejemplar de diseño popular está conformado por una estructura en forma de "S", a base de concreto armado y terminada en color blanco; este mobiliario es prefabricado artesanalmente, por lo que es común que presenten diferencias singulares entre sí.

Actualmente, en un mundo que se debate entre lo local y global, el diseño de mobiliario urbano deriva de una tendencia de homogeneización excesiva, provocada por la 
oferta de los fabricantes y los intereses de los gestores que buscan comprar siempre al mejor precio (Fernández, 2004). Por ello, es importante renovar el mercado a través de propuestas que propicien la imaginación, la improvisación, el mejoramiento y la readaptación de los elementos insertos en el espacio público.

Por otra parte, es importante entender como el diseño, se ha desarrollado en las últimas décadas para establecer una propuesta o proceso creativo que responda a los requerimientos de las demandas contemporáneas.

En este mismo sentido a lo largo del tiempo, el diseño ha transformado la producción material provocada por la variación de las materias primas, tendencias y tecnologías. Lo anterior ha sido la pauta para la implementación de diferentes procesos.

Llegados los años setenta con los materiales y técnicas nuevos, el diseño interactúa con la tecnología en distintos niveles de producción y mercadeo; propiciando que a finales del S.XX, la transformación del propio proceso de diseño cambie de método de fabricación de producción en serie a producción en serie flexible. Este último, permite que el diseño no se rija por una sola tendencia, sino que adopte otros métodos de fabricación con la capacidad de adaptarse a las cambiantes pautas del consumo global (Sparke,2010).

Este estudio hibrida las habilidades tácticas de la producción artesanal con la implementación de nuevas tecnologías en los procesos de diseño y fabricación, para aproximarse a nuevos efectos diferenciadores, nuevas sensaciones, nuevas combinaciones, y con ello generar nuevos lugares, una cultura propia, singular, que pueda tener su reflejo en el mobiliario de sus ciudades (Fernández, 2004).
Populus, es un vocablo latín, plural, cuyo significado se referencia a la comunidad humana, al pueblo, la gente, la nación, la etnia; incluso en su traducción más literal significa "amigos" (Villalba,1995). Tal nomenclatura enfatiza el diálogo existente entre el objeto y el usuario para formar parte del imaginario popular.

\section{METODOLOGÍA}

El proyecto Populus, hibrida diversas herramientas, provenientes de la investigación documental y de campo, el prototipado rápido, la fabricación digital, entre otros, constituyendo así, su propia estructura metodológica. La cual, propicia la retroalimentación constante con la experimentación y la fabricación de modelos a escala física y digital, así mismo, permite la observación de fallas y aciertos, mejorando dichos modelos, en un proceso evolutivo e iterativo que permita entender la indeterminación del objeto, anticipándose a su construcción a escala usuario.

A partir de una estructura horizontal, con el fin de generar un proceso cíclico que promueva la constante retroalimentación en el desarrollo del diseño. La metodología se compone de siete fases: (1) Investigación Documental y de Campo, (2) Definición del Objeto de Estudio, (3) Primeras Aproximaciones al Objeto, (4) Concepto de Diseño, (5) Prototipado Rápido y Fabricación Digital, (6) Proyección de fabricación en Serie y (7) Visualización y Especulación del Objeto (Figura 2).

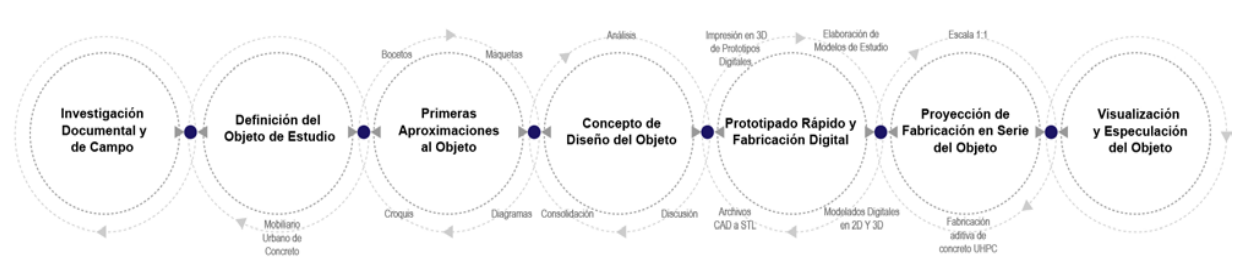

Figura 2: Mapa mental que muestra la estructura metodológica empleada en el proceso de diseño, donde se distinguen las actividades esenciales desarrolladas en cada fase, así como la vinculación entre sí, como parte de un ciclo que promueve el análisis, la revisión y la evaluación continua de la información y del propio objeto en estudio.

Por lo anterior surge Populus, una investigación en curso en la era de la cultura maker (cultura hacedora) y el do it yourself (hágalo usted mismo); con el objetivo de desarrollar un proceso de diseño, basado en la aplicación de herramientas derivadas del prototipado rápido y la fabricación digital, para explorar posibles aspectos materiales y funcionales, de una propuesta de diseño de mobiliario urbano de concreto, que responda a la resignificación del diseño popular y a la solución de la escasez de equipamiento funcional, cómodo y resistente a la degradación. 


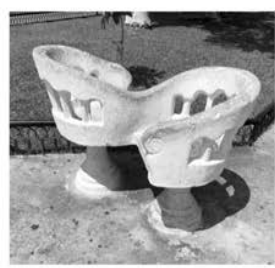

01

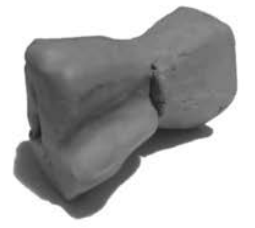

04

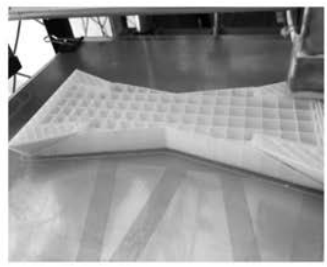

07

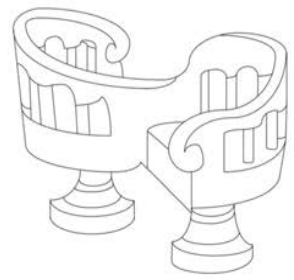

02

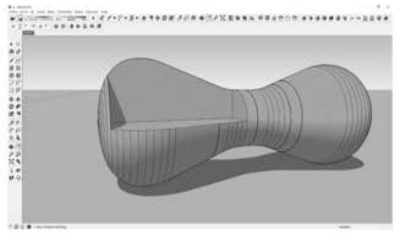

05

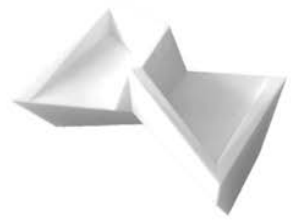

08

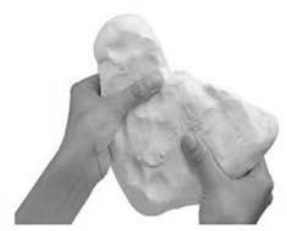

03

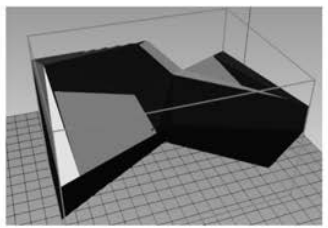

06

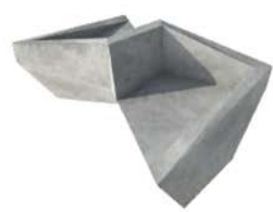

09

01-02 Investigación / 03-04 Modelado Análogo / 05 Modelado Digital / 06 Migración Archivos CAD-STL / 07 Fabricación Digital por Manufactura Aditiva / 08 Prototipo Rápido / 09 Visualización y Especulación del Objeto

Figura 3: Diagrama que muestra el proceso de diseño ilustrado de cada una de las fases desarrolladas a lo largo de la metodología, donde se percibe cronológicamente desde los elementos básicos de entrada como la investigación, hasta los elementos finales de salida con la visualización y especulación del objeto.

\section{RESULTADOS}

Populus parte de la exploración formal, material y ergonómica, con la intención de releer, reinterpretar y resignificar el diseño popular implícito de la mencionada Silla Confidentes. Así mismo, se valida que los materiales más comunes que conforman el mobiliario urbano son: hierro fundido, aluminio, madera, acero galvanizado o inoxidable, plásticos y particularmente concreto hidráulico; material que ha estado vigente y en constante evolución, al ofrecer posibilidades alternas cuando se mezcla con diversos agregados, fibras y aditivos que permiten mejorar drásticamente sus características y maleabilidad, permitiendo nuevas posibilidades de uso.

Tras definir el concepto de diseño, y determinar los alcances de este primer estudio, el proceso de prototipado comienza con el análisis de las diversas ideas, realizando bocetos y croquis que permiten tener una primera aproximación al objeto. Se procede a la fabricación de maquetas de estudio volumétricas a escala, que permiten sintetizar la geometría del caso análogo citado 
anteriormente, empleando un material que permite modificar la plástica del objeto, como lo es la plastilina, elemento plástico utilizado para moldear, ya que posee algunas condiciones de maleabilidad similares a las que responden algunas propiedades del concreto.

Con los primeros esbozos y los primeros prototipos a base de plastilina, se hace una exploración de la tridimensionalidad del objeto, mediante el trazado digital integrado por entidades geométricas vectoriales y volumétricas, con el empleo de herramientas de diseño asistido CAD (Computer Aided Design), haciendo una manipulación gráfica, directa e intuitiva. Esta exploración permite analizar de una manera más amplia aspectos formales, funcionales y estructurales; así como la aproximación sobre la ergonomía y estética del objeto.

Posteriormente, los archivos CAD tridimensionales, son exportados o convertidos a la extensión denominada STL (STereoLithography - Standard Triagle or Tessellation Language) esterolitografía mediante un software $3 D$. Una vez procesados los archivos STL, bajo el método de manufactura aditiva (o fabricación por adición), se emplea una impresora 3D de FDM (ó MDF-Modelado de Deposición Fundida), para fabricar un modelo de extrusión plástica, usando un termoplástico, constituido por un polímero de clase PLA (ácido poliláctico, de $1.75 \mathrm{~mm}$ de diámetro). De esta manera se obtiene un prototipo rápido tridimensional, mismo que será susceptible de revisión e identificación de mejoras como un parte de proceso cíclico.

Así mismo, se desarrollan visualizaciones, a base de renders y collages digitales, para exhibir escenarios posibles, especulando sobre las diversas posibilidades de uso, e integración de las piezas con múltiples entornos urbanos, y la interacción con las personas.

Con este estudio se valida como la fabricación digital, resulta clave en los procesos de diseño de un objeto, gracias al empleo de herramientas de simulación, visualización en $3 \mathrm{D}$, análisis y colaboración con el objetivo de definir cualidades del producto y la fabricación simultánea de prototipado rápido. Así mismo, permite asociar, visualizar y gestionar cambios de información sobre productos, procesos y/o recursos, bajo una una perspectiva coherente y completa ya no solo del diseño, sino también de la futura producción en serie.

Los parámetros para el diseño de los modelos Populus seleccionados, derivan de un prisma rectangular, el cual ha sido sometido a una serie de cortes y sustracciones que permiten resaltar la geometría del objeto, a la par de restar masa o volumen, y consecuentemente evitar el exceso de peso y sobredimensionamiento, al generar cavidades que permitan ahorrar materia prima en la elaboración escala 1:1. El respaldo en forma de "S", funge como soporte estructural, sin llegar a aislar los asientos, favoreciendo la comunicación entre usuarios y potencializando las posibilidades de uso (Figura 4).

Las piezas de prototipado rápido generadas a partir de la metodología propuesta, han permitido un proceso cíclico de constante análisis, discusión y evaluación, de las diversas cualidades físicas y materiales, así como medir la factibilidad de crecimiento o proliferación del objeto para finalmente seleccionar un prototipo, cuya forma responde a una serie de planos inclinados dispuestos a diversos

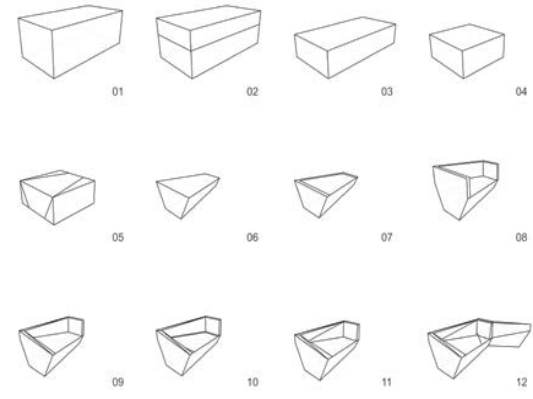

Figura 4: Proceso de Diseño Digital que muestran diagramas de la evolución morfológica del prototipo, donde se procuró estilizar la geometria y reconfigurar sus aristas; procurando la menor masa posible.

ángulos que re-sintetizan geométricamente la Silla Confidentes.

La proliferación señalada se manifiesta al idear Populus como un ente que proyecta factibilidad de crecimiento en sentido longitudinal (Eje Y). De esta manera, es posible crear Populus Duo y Populus Múltiple (Figura 5 y 6 ), el primer modelo es una banca con medidas aproximadas de $180 \times 120 \times 86 \mathrm{~cm}$ con capacidad de alojar a dos personas; mientras que el segundo modelo, con medidas aproximadas de $240 \times 120 \times 86 \mathrm{~cm}$ aloja hasta cuatro personas. En ambos casos se aplicarán la misma lógica técnica, funcional y constructiva para su producción futura.
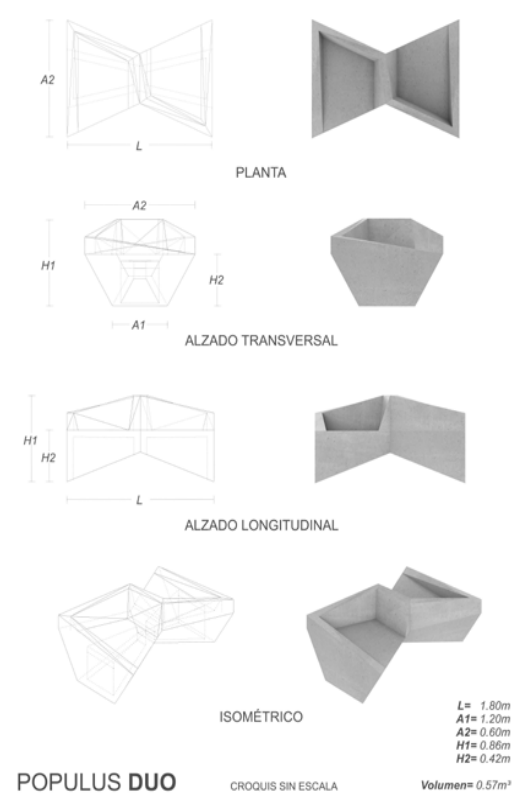

Figura 5: Diagrama que muestra las diversas vistas en planta y alzados longitudinales y transversales del prototipo seleccionado versión: Populus Duo. 

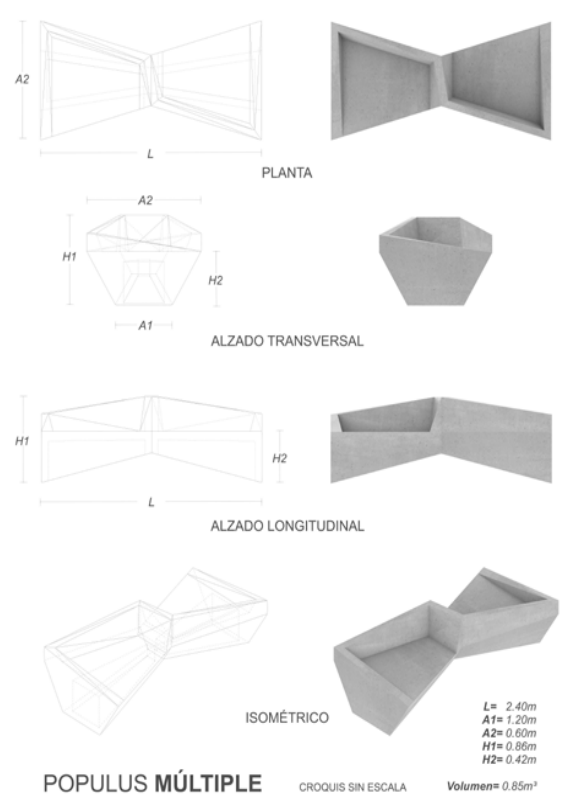

Figura 6: Diagrama que muestra las diversas vistas en planta y alzados longitudinales y transversales del prototipo seleccionado versión: Populus Múltiple

\section{DISCUSIÓN}

Este estudio, contribuye a consolidar un proceso de diseño y fabricación de un mobiliario urbano que revalora al diseño popular de las ciudades, para formar parte del imaginario colectivo; empleando la tecnología al alcance, la vanguardia industrial y la arquitectura contemporánea con un vocabulario universal, consolidando un elemento singular de un alto valor añadido.

A partir de la investigación realizada para identificar la naturaleza e importancia del mobiliario urbano, se distingue que estas micro-arquitecturas son esenciales para la conformación del espacio público de calidad en la ciudad contemporánea. Se observa cómo el diseño y la oferta del mobiliario responden a intereses específicos, abriendo áreas de oportunidad para la exploración e innovación.

Simultáneamente, se hace evidente la resiliencia innata de diseñador, con la optimización de recursos y herramientas para solucionar entre otras cosas, la escasez de dicho equipamiento. Se identifica la Silla Confidentes, como un componente urbano de diseño popular, que sirve de análisis al presente trabajo, al estar fabricada en concreto, y al ser un elemento que se enraíza a la cultura local; para posteriormente desarrollar una síntesis y re-interpretación no solamente del diseño, sino también de su posible fabricación futura.

A través de la aplicación de herramientas que brindan el prototipado rápido y la fabricación digital, ha sido posible la creación de modelos a escala, tanto análogos como digitales, para evaluar diversas características físicas y materiales (funcionalidad, ergonomía, resistencia e integración a múltiples contextos); para aproximarse a soluciones de mobiliario urbano más eficientes y sustentables.

Este estudio en el futuro, optará por emplear el concreto como materia prima en el proceso de fabricación, gracias a la alta durabilidad y resistencia, así como la gran disponibilidad al poder ser fabricado en cualquier parte del mundo, contribuyendo de esta manera a la optimización de recursos y a la reducción de la huella de carbono, así mismo, sus propiedades estéticas permiten la innovación y flexibilidad en el diseño (Ceballos,2016)

Por lo anterior, el proyecto Populus buscará reproducir un prototipo en escala 1:1, empleando una variedad de concreto denominada UHPC (Ultra-High Performance Concrete o Concreto de Ultra-Alto Desempeño), el cual diversos estudios exponen como este material, al integrar en su mezcla fibras de acero y polipropileno, pueden modificar satisfactoriamente sus capacidades mecánicas (Christ,et al,2019), haciendo posible con ello su fabricación bajo el método de manufactura aditiva.

De igual manera, y como respuesta alterna, la presente investigación también podrá guiarse en torno a los materiales empleados, vinculando la tecnología y el oficio disponibles para la producción en serie. Partiendo de la fabricación del molde (acero, madera, silicón), hasta la formulación específica de las materias primas (agua, áridos o agregados, cemento, colorantes, armadura, fibras de refuerzo y aditivos químicos) y acabados finales (crudo o salido del molde, decapado, pulido), entre otros.

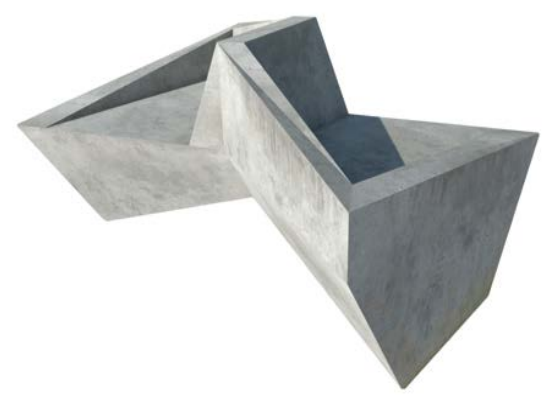

Figura 7: Ilustración que muestra la visualización del prototipo a escala de usuario del modelo Populus Duo. 


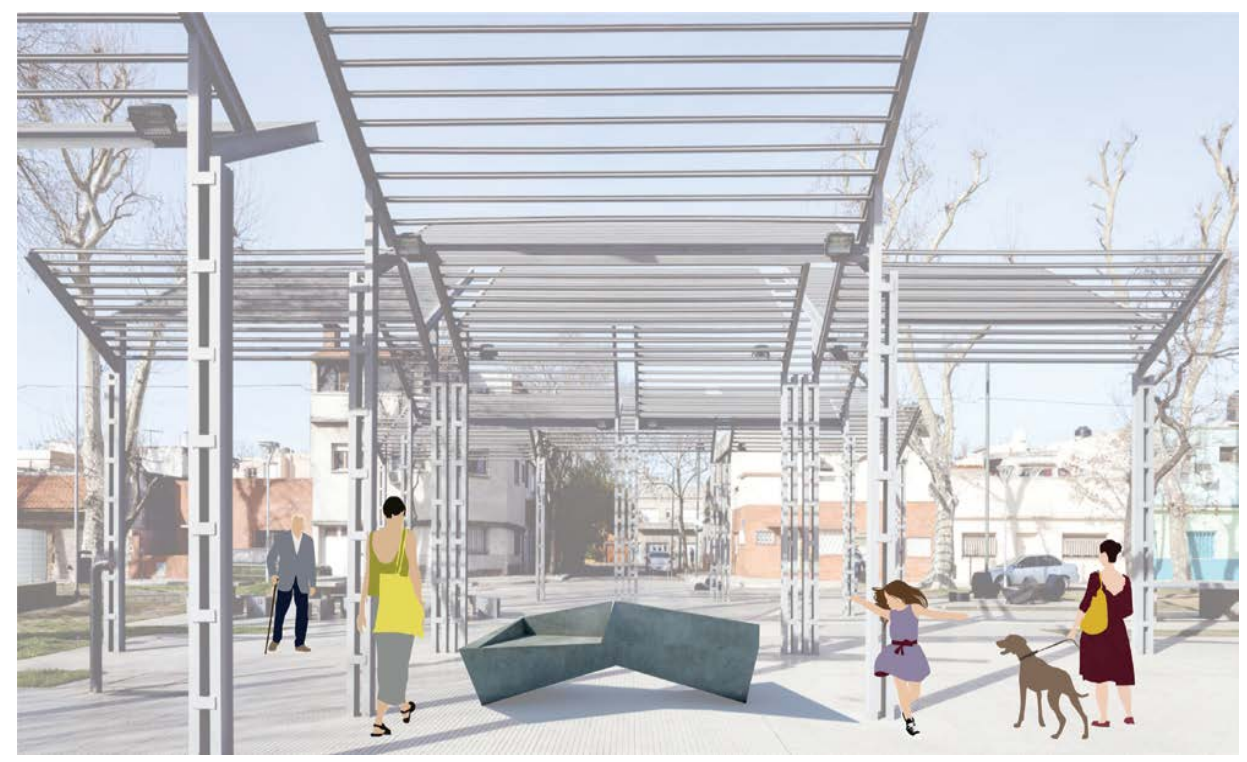

Figura 8: Especulación gráfica de inserción en el espacio público de la banca Populus.

\section{REFERENCIAS}

Ceballos, A. (2016). El Concreto, Material Fundamental para la Infraestructura, Construcción y Tecnología en Concreto,Vol. 6 , No11,24-25

Christ, Pacheco, Ehrenbring, Quinino, Mancio, Muñoz, Tutikian (2019). Estudio del comportamiento mecánico del hormigón de ultra-altas prestaciones (UHPC) reforzado con fibras híbridas y con consumo reducido de cemento. Revista Ingeniería de Construcción, Vol 34, No.2, 159-168.

Del Real, P. (2013). El Mobiliario Urbano como Objeto de Uso Público: Implicaciones para su Diseño. Trilogía Ciencia Tecnología Sociedad, vol.25, no.35.

Escobar, A. (2016). Autonomía y diseño: la realización de lo comunal. Popayán, Colombia. Universidad del Cauca.

Fernández, M. (2004). Mobiliario urbano: un elemento diferenciador en las ciudades. QEJ. Bricojardinería y paisajismo: Revista profesional de distribución en horticultura ornamental y jardinería, No.125, 10-16

Manzini, E. (2015). Design, when everybody designs: an introduction to design for social innovation. Cambridge, USA. MIT Press

Mallet, A. (2018). Silla Mexicana, México: Arquine,

Mendoza, M (2016). Tradiciones: Las Sillas Confidentes. https://yucatantoday.com/las-sillas-confidentes/

Robles, A. (2016). Fabricación digital en arquitectura: técnicas, recursos y conocimiento material. RDU Revista Digital Universitaria UNAM, 17 (8), 3-12.

Sparke, P. (2010). Diseño y cultura una introducción desde 1900 hasta la actualidad, Barcelona, España:Gustavo Gili

Villalba, J (1995). Estudio Léxico del Pueblo en Táctico: Vulgus,Plebs,Populus. AEF XVIII, 533-549, Recuperado de http://dehesa.unex.es/bitstream/handle/10662/1930/02108178_18_533.pdf?sequence=1\&isAllowed $=y$ 